\title{
Compatibilidade fenotípica entre cultivares de pereiras europeias e porta-enxertos de marmeleiro
}

\author{
Phenotypic compatibility among european pear cultivars and quince rootstocks
}

\author{
Bruno Dalazen Machado ${ }^{I}$ Maicon Magro ${ }^{I}$ Leo Rufato ${ }^{I}$ Amauri Bogo ${ }^{\text {I* }}$ \\ Aike Anneliese Kreztschmar ${ }^{I}$ Fabiano Simões ${ }^{I I}$
}

\section{RESUMO}

A cultura da pereira (Pyrus communis L.) possui grande potencial de expansão no sul do Brasil devido às condições climáticas e de solo. A carência de informações a respeito da(s) melhor(es) combinação(ões) de cultivares 'copa de pereiras europeias' e porta-enxertos de marmeleiro em relação à compatibilidade de enxertia é um dos fatores que tem limitado o cultivo de pera no país. A utilização de portaenxertos de marmeleiros apresentam as vantagens de redução do vigor e rápida entrada em produção, porém, quando enxertados com algumas cultivares de 'pereira europeia', pode acarretar incompatibilidade de enxertia. $O$ trabalho foi realizado com o objetivo de avaliar fenotipicamente a compatibilidade morfológica entre cultivares 'copa de pereiras europeias'e porta-enxertos de marmeleiro através das variáveis de crescimento das plantas, como o incremento de diametro ( $\mathrm{mm}$ ) do tronco da cultivar e do porta-enxerto; diferença de diâmetro entre a cultivar e o porta-enxerto; incompatibilidade 'translocada'; incompatibilidade 'localizada'; e análise da conexão vascular no ponto de enxertia pela imersão em solução corante de Ácido Fuccínico 0,08\%. Os experimentos foram conduzidos em pomares de pereiras europeias na região de Urupema, estado de Santa Catarina, durante os ciclos de cultivo 2011/12, 2012/13 e 2013/14. As combinações de cultivares de pereiras europeias e porta-enxertos avaliados foram a 'Abbè Fetel'/'Adams', 'Decana du Comice'/'Adams', 'Clapp's Favourite'/EMA', 'Rocha'/Adams', 'Packham's Triumph'/EMA' e 'Santa Maria'/'Adams'. A combinação 'Clapp's Favourite'/'EMA' apresentou incompatibilidade do tipo 'localizada' pela descontinuidade vascular na região de união do enxerto, impedindo a passagem do corante. As combinações 'Rocha'/Adams' e 'Abbè Fetel'/'Adams' não apresentaram incompatibilidade de enxertia. Nessas combinações, foram observadas união vascular contínua entre as cultivares e os porta-enxertos. As combinações 'Santa Maria'/'Adams', 'Decana du Comice'/'Adams'e 'Packham's Triumph'/EMA' apresentaram boa união na zona de enxertia pela continuidade da linha de união na casca e no lenho. A combinação 'Clapp's Favourite'/'EMA', devido às regiões de incompatibilidade encontradas, não é uma combinação recomendada nas condições experimentais avaliadas. As combinações 'Abbè Fetel'/'Adams'e 'Rocha'/Adams' são as mais indicadas para plantio, devido à total compatibilidade encontrada entre a cultivar e o porta-enxerto. As combinações 'Santa Maria'/'Adams', 'Decana du Comice'/'Adams' e 'Packham's Triumph'/EMA' podem ser recomendadas, devido à compatibilidade parcial de enxertia, sendo indicadas para alta e super densidades de plantio.

Palavras-chave: Pyrus communis, união vascular, níveis de compatibilidade, vigor, incompatibilidade 'localizada', incompatibilidade 'translocada'.

\section{ABSTRACT}

The pear (Pyrus communis L.) crop offers an expansive market opportunity in southern Brazil, because of amicable climatic and soil conditions. However, the lack of studies on compatibility of european pear cultivars and rootstocks is one of the limiting factors on the development of the pear crop in southern Brazil. The use of quinces as rootstocks has the advantages of vigor reduction and earlier bearing trees. However, some of these combinations can present some incompatibility graft. The objective of this study was to evaluate phenotypicaly the morphological compatibility among european pear cultivars and quince rootstocks. The evaluated variable were the section increment of cultivars and rootstocks trunk diameter at the graft union; the diameter difference between scion and cultivars and rootstocks; 'translocated' incompatibility; 'located' incompatibility; and the vascular connection of the graft union by immersion of the base of the plants (under the graft union) in a $0.08 \%$ Fuccinic acid solution. The experiments were conducted in an european pear orchards in the region of Urupema municipality, state of Santa Catarina, during the 2011/12, 2012/13 and 2013/14 growing seasons. The european pear cultivars and rootstocks evaluated were:

'Programa de Pós-graduação em Produção Vegetal, Universidade do Estado de Santa Catarina (UDESC), Avenida Luis de Camões, 2090, 88520-000, Lages, SC, Brasil. E-mail: amauribogo@udesc.br. "Autor para correspondência.

"Departamento de Agronomia, Universidade Estadual Rio Grande do Sul (UERGS), Vacaria, RS, Brasil. 
'Abbé Fetel'/'Adams', 'Decana du Comice'/Adams', 'Clapp's Favourite'/'EMA', 'Rock'/'Adams', 'Packham's Triumph'/'EMA' and 'Santa Maria'/'Adams'. 'The Clapp's Favourite'/'EMA' combination showed a 'located incompatibility by descontinued vascular graft region, preventing the dye translocation. 'The Rocha'/'Adams' and 'Abbè Fetel'/'Adams' combinations did not present graft incompatibility, showing continued vascular union and no vascular disruption between cultivars and rootstocks. The 'Santa Maria'/'Adams', 'Decana du Comice'/'Adams' and 'Packham's Triumph'/EMA' combinations showed a good graft union region with clear and continued bark and wood bond lines. The 'Clapp's Favourite'/'EMA' combination showed high incompatibility and is not recomented for the experimental conditions evaluated. The 'Abbè Fetel'/'Adams' and 'Rocha'/'Adams' combinations showed the highest graft compatibility among cultivars and rootstocks and are the best recomended combinations. Additionly, the 'Santa Maria'/'Adams', 'Decana du Comice'/'Adams' and 'Packham's Triumph'/'EMA' combinations showed partial compatibility and can eventually be indicated for high and super plant density when 'Abbè Fetel'/'Adams' and 'Rocha'/'Adams' combinations are not available.

Key words: Pyrus communis, vascular union, compatibility levels, vigour, located incompatibility, translocated incompatibility.

\section{INTRODUÇÃO}

O Brasil ocupa o quadragésimo quinto lugar na produção de pera (Pyrus communis L.), com produções inferiores a países com extensões territoriais menores do que a grande maioria dos estados brasileiros (TECCHIO et al., 2011). As áreas de produção nacional estão concentradas, principalmente, nos estados da região sul e sudeste, com produção anual de aproximadamente 20 toneladas e produtividade média de $11 \mathrm{t} \mathrm{ha}{ }^{-1}$ (FAO, 2013). Em virtude deste cenário, a produção brasileira é insuficiente para atender a demanda interna, que consome aproximadamente um volume oito vezes maior (TECCHIO et al., 2011), fazendo com que a cultura represente uma excelente oportunidade de mercado para os produtores nacionais (FACHINELLO et al., 2011).

Dentre as dificuldades de expansão da cultura da pereira, pode-se citar a baixa qualidade da pera produzida no Brasil, principalmente pela limitação de cultivares adaptadas às regiões potencialmente produtoras, razão pela qual onera o baixo rendimento de pereiras no país (PIO et al., 2008). De acordo com GIACOBBO et al. (2007), além da falta de informações sobre cultivares e portaenxertos, outro aspecto que tem desfavorecido a produção de peras no país é a falta de conhecimento sobre a melhor combinação de cultivar e porta-enxerto que não apresentam problemas de incompatibilidade de enxertia.
Os marmeleiros vêm sendo utilizados como porta-enxertos preferenciais para a pereira, com $\mathrm{o}$ intuito de proporcionar plantas de pequeno porte $\mathrm{e}$ rápida frutificação, além de conferir uniformidade aos pomares (MILOSEVIC \& MILOSEVIC, 2011). Apesar de o marmeleiro proporcionar uma considerável redução de vigor às plantas e precocidade de produção, no entanto, um fator que tem prejudicado a expansão do marmeleiro como porta-enxerto para a cultura da pereira europeia é a incompatibilidade morfológica entre ambos, fazendo com que haja maior dificuldade de introdução de novas variedades de porta-enxertos (PINA \& ERREA, 2009).

Apesar dos eventos envolvidos na enxertia serem conhecidos, o mecanismo pelo qual a incompatibilidade se expressa ainda não está definido, sendo necessários estudos para explicar este fenômeno. MOSSE (1962) dividiu a incompatibilidade em 'translocada' e 'localizada'. A 'translocada' ocorre quando algum fator ocasional, como uma toxina, é transportada de um componente do enxerto para o outro e a inserção de um filtro mutuamente compatível não supera essa incompatibilidade. Já a 'localizada' ocorre na interface do enxerto e requer o contato entre os dois componentes do enxerto, uma vez que a inserção de um filtro mutuamente compatível supera a incompatibilidade.

Nesse contexto, o objetivo do trabalho foi estudar fenotipicamente a compatibilidade entre cultivares de pereiras europeias e porta-enxertos de marmeleiro, através de variáveis de crescimento das plantas e análise da conexão vascular no ponto de enxertia.

\section{MATERIAL E MÉTODOS}

Os experimentos foram conduzidos em pomares comerciais de Urupema, estado de Santa Catarina, durante os ciclos de cultivo 2011/12,2012/13 e $2013 / 14$. A região $\left(28^{\circ} 17^{\prime} 38^{\prime \prime} \mathrm{S} / 49^{\circ} 55^{\prime} 54^{\prime \prime} \mathrm{W}\right)$ está localizada a aproximadamente $1425 \mathrm{~m}$ de altitude acima do nível do mar e clima mesotérmico úmido Cfb-A, de acordo com a classificação de Köppen (PEEL et al., 2007), sem estação seca, com verão ameno e precipitação distribuída durante todo o ano, com índice pluviométrico médio de $1789 \mathrm{~mm}$ anual e temperatura média anual de $14^{\circ} \mathrm{C}$ (EPAGRI, 2013). O solo da região é do tipo nitossolo, com textura franco argilosa, com teores altos de matéria orgânica (6\%) e argila $(60 \%)$.

Os pomares foram implantados em agosto de 2007. O plantio foi realizado com mudas préformadas através do método de enxertia por garfagem 
e o sistema de condução adotado foi o Líder Central. A área experimental foi implantada com espaçamento de $1 \mathrm{~m}$ entre plantas e $4 \mathrm{~m}$ entre linhas, correspondendo a uma densidade de plantio de 2500 plantas $\mathrm{ha}^{-1}$.

As combinações cultivar copa de pereiras europeias e porta-enxertos de marmeleiro utilizadas foram: 'Abbè Fetel'/'Adams', 'Decana du Comice'/'Adams', 'Clapp's Favourite'/'EMA', 'Rocha'/'Adams', 'Packham's Triumph'/EEMA' e 'Santa Maria'/'Adams'. Para a avaliação da compatibilidade de enxertia, foram avaliadas variáveis de crescimento de plantas e análise de conexão vascular no ponto de enxertia. As variáveis de crescimento de plantas avaliadas foram: a) incremento de diâmetro (mm) do tronco da cultivar (IDC) e do porta-enxerto (IDPE); b) diferença de diâmetro entre a cultivar e o porta-enxerto; c) incompatibilidade 'translocada'; d) incompatibilidade 'localizada'.

Para a avaliação das variáveis ' $a$ ' e ' $b$ ', as plantas foram marcadas com tinta plástica cinco centímetros abaixo e acima do ponto de enxertia. As medidas foram realizadas na mesma zona de leitura, durante os três anos de avaliação, com auxílio de paquímetro digital. Com as medidas, calculou-se a diferença entre o diâmetro do tronco das cultivares copa e dos porta-enxertos. A incompatibilidade 'translocada' foi avaliada pelo teor de clorofila médio (SPAD) de 10 folhas por planta ${ }^{-1}$ (uma folha por quadrante) de cada tratamento com cinco plantas por parcela, totalizando 30 folhas por parcela. As folhas estavam em pleno desenvolvimento vegetativo, com tonalidade variava de verde-amarelada (clorótica) à verde-escura, a aproximadamente 150 dias após a plena floração. A leitura foi realizada com medidor portátil de clorofila SPAD-502, adotando como critério folhas localizadas no terço médio da planta, inteiras e sadias, com os dados expressos em unidades SPAD. A incompatibilidade 'localizada' foi avaliada pela análise anatômica interna e externa da região de união dos enxertos 8 anos após o enxerto, de acordo com a classe de notas (A, B, C, D e E) segundo MOSSE \& HERREO (1951), sendo: A: união perfeita, onde a linha de união não é visível; B: boa união, a linha de união na casca e no lenho são contínuas, embora, no lenho, seja muitas vezes visível devido a excessiva formação de raias; $C$ : união com descontinuidade na casca, os tecidos da casca do porta-enxerto e da cultivar são separados por uma camada marrom escura com aparência de cortiça; D: uniões com descontinuidade vascular, os tecidos do lenho do porta-enxerto e da cultivar são separados, por outro lado, os tecidos da casca seguem como na classe $\mathrm{C}$; e $\mathrm{E}$ : quando ocorre quebra da união no pomar ou viveiro. As classes A, B e C são consideradas compatíveis, pois não prejudicam a resistência mecânica, entretanto, as classes D e E são incompatíveis, pois podem romper-se por dano mecânico ou ação do vento.

Para a observação da conexão vascular no ponto de enxertia das diferentes combinações de cultivares de pereiras europeias e porta-enxertos de marmeleiro, foi realizado o corte de tronco $10 \mathrm{~cm}$ acima e abaixo do ponto de enxertia, sendo imediatamente imersos em água, a fim de evitar o processo de cavitação. No fitotron, os cortes de troncos receberam gotejamento de água ultrapura por um período de uma hora para expulsão de ar dos vasos do xilema e floema, evitando a cavitação seguida do gotejamento de solução corante Ácido Fuccínico $0,08 \%$ por uma hora para coloração dos vasos na região do enxerto. Após a coloração, incisões transversais foram realizadas para a visualização da continuidade dos vasos por meio do acúmulo do corante na região de enxertia. As regiões pigmentadas com o corante foram fotografadas com câmara fotográfica Fugifilm Finepix S3300.

O delineamento experimental utilizado foi o de blocos ao acaso, com três repetições e cinco plantas por parcela. Os resultados foram submetidos à análise de variância (ANOVA) ao teste de comparação múltipla de médias, utilizando-se Duncan a $5 \%$ de probabilidade de erro para determinar as diferenças de compatibilidade entre as combinações de cultivares de pereira e porta-enxertos de marmeleiro.

\section{RESULTADOS E DISCUSSÃO}

O maior vigor das plantas, caracterizado pelo IDPE (incremento do diâmetro do tronco do porta-enxerto) e IDC (incremento do diâmetro do tronco da cultivar), foi observado na combinação 'Abbè Fetel'/'Adams' (Tabela 1). Este maior vigor pode estar associado à compatibilidade na zona de enxertia, pois a linha de união na casca e no lenho estavam contínuas, permitindo a passagem do corante ácido fuccínico nos feixes vasculares, demonstrando um fluxo contínuo entre os vasos condutores do portaenxerto com a cultivar (Figura 1). Este fluxo contínuo influencia o crescimento pelo efeito na translocação de nutrientes e fotoassimilados das raízes para os ramos e dos ramos para as raízes (RODRIGUES et al., 2004; ZARROUK et al., 2006).

A combinação 'Clapp's Favourite'/'EMA' obteve o maior valor na diferença de diâmetro entre o porta-enxerto e a cultivar, quando comparado às demais combinações (Tabela 1). Plantas com 
Tabela 1 - Análise do incremento no diâmetro do tronco da cv. copa (IDC), incremento no diâmetro do porta-enxerto (IDPE) e diferença de diâmetro entre o porta-enxerto de marmeleiro e a copa $(\mathrm{PE}-\mathrm{C})$ das diferentes combinações de cultivares de pereiras europeias durante os ciclos de cultivo 2011/12, 2012/13 e 2013/14, Urupema, SC.

\begin{tabular}{|c|c|c|c|c|}
\hline \multicolumn{2}{|c|}{ 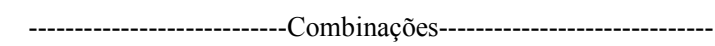 } & \multirow{2}{*}{$\frac{\mathrm{IDC}(\mathrm{mm})}{21,33 \mathrm{a}}$} & \multirow{2}{*}{$\frac{\text { IDPE }(\mathrm{mm})}{24,00 \mathrm{a}}$} & \multirow{2}{*}{$\frac{\mathrm{PE}-\mathrm{C}(\mathrm{mm})}{2,67 \mathrm{c}}$} \\
\hline 'Abbè Fetel' & 'Adams’ & & & \\
\hline 'Clapp's Favourite’ & 'EMA' & $5,67 \mathrm{e}$ & $11,00 \mathrm{e}$ & $5,33 \mathrm{a}$ \\
\hline 'Decana du Comice' & ‘Adams’ & $10,67 \mathrm{~d}$ & $15,00 \mathrm{~cd}$ & $4,33 \mathrm{~b}$ \\
\hline 'Packham's Triumph' & 'EMA' & $13,33 \mathrm{~cd}$ & $15,33 \mathrm{~cd}$ & $2,00 \mathrm{~d}$ \\
\hline 'Rocha' & 'Adams' & $16,00 \mathrm{~b}$ & $17,67 \mathrm{~b}$ & $1,67 \mathrm{e}$ \\
\hline 'Santa Maria' & 'Adams' & $13,28 \mathrm{~cd}$ & $14,67 \mathrm{~cd}$ & $1,39 \mathrm{f}$ \\
\hline CV $(\%)$ & & 11,06 & 10,10 & 5,08 \\
\hline
\end{tabular}

*Médias seguidas pela mesma letra na coluna não diferem estatisticamente entre si pelo teste de Duncan a 5\% de probabilidade de erro.

sintomas de incompatibilidade apresentam as maiores diferenças de diâmetro da copa em relação ao portaenxerto, corroborando RODRIGUES et al. (2004), que descrevem a ocorrência deste crescimento no ponto de enxertia em decorrência das diferentes taxas de divisão celular entre a região do câmbio do portaenxerto e da cultivar, diferindo na velocidade de formação do xilema e do floema, o qual definiu como sintoma de incompatibilidade de enxertia.

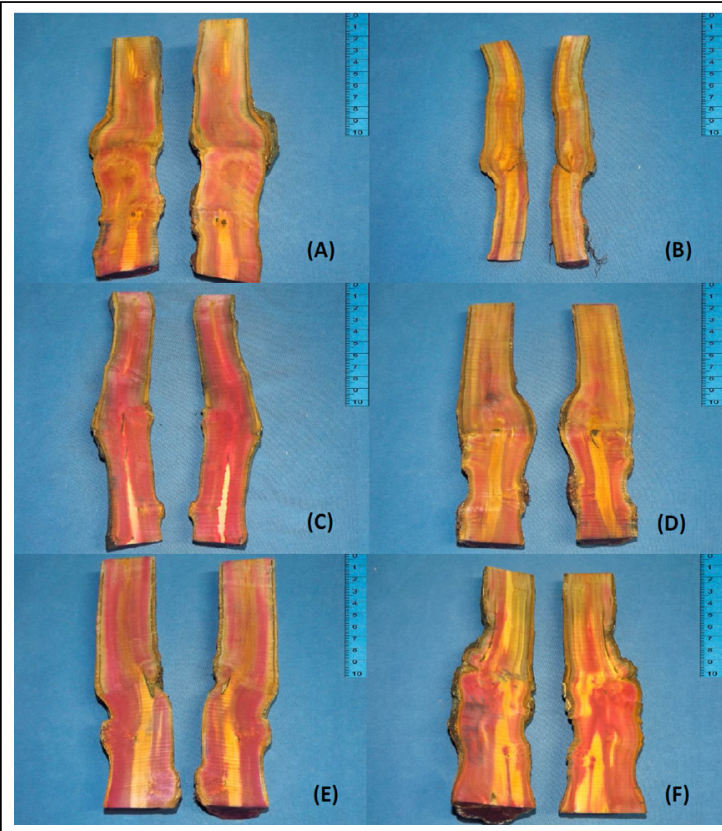

Figura 1 - Corte macroscópico longitudinal interno para observar a conexão vascular no ponto de enxertia das combinações de cultivares de pereiras europeias e porta-enxertos de marmeleiro 'Decana du Comice'/‘Adams' (A), 'Clapp's Favourite'/'EMA' (B), 'Rocha'/'Adams' (C), 'Packham's Triumph'/'EMA' (D), 'Abbè Fetel'/'Adams' (E) e 'Santa Maria'/'Adams' (F), oito anos após a enxertia em Urupema,SC.
A combinação 'Clapp's Favourite'/'EMA' apresentou o menor índice SPAD (34) nos teores de clorofila, indicando a ocorrência de incompatibilidade do tipo 'translocada' (Tabela 2). Já as combinações 'Packham's Triumph'/'EMA', 'Abbè Fetel'/'Adams' e 'Rocha'/'Adams' apresentaram os maiores índices SP de 46, 45,4 e 44,8 de teor de clorofila na folha, respectivamente, demonstrando ausência de incompatibilidade do tipo 'translocada', pois quanto maior o grau de incompatibilidade 'translocada' menor o índice SPAD (Tabela 2) (ZARROUK et al., 2006).

A combinação 'Clapp's Favourite'/'EMA' apresentou incompatibilidade 'localizada' (Tabela 2). Pela avaliação macroscópica anatômica do ponto de união dos enxertos (Figura 2), não permitindo a passagem do corante ácido fuccínico, classificando como incompatível da classe D (Tabela 2) (MOSSE \& HERREO (1951). A combinação 'Clapp's Favourite'/'EMA' apresentou baixa resistência mecânica, morte de plantas (dados não apresentados) e um crescimento excessivamente reduzido, sendo considerados aspectos negativos em nível de campo.

As combinações 'Rocha'/Adams' e 'Abbè Fetel'/'Adams' apresentam uma união vascular contínua perfeita entre os portaenxertos e as cultivares, sendo consideradas classe A da escala de MOSSE \& HERREO (1951) (Figura 1). A observação dos cortes longitudinais do tronco na região do enxerto das combinações 'Rocha'/'Adams' e 'Abbè Fetel'/'Adams' possibilitaram a visualização da passagem do corante ácido fuccínico pelos feixes vasculares, demonstrando um fluxo contínuo entre os vasos condutores dos portaenxertos com as cultivares (Figura 1).

As combinações 'Santa Maria'/'Adams', 'Decana du Comice'/'Adams' e 'Packham's 
Tabela 2 - Incompatibilidade 'translocada' e 'localizada' entre as diferentes combinações de cultivares de pereiras europeias e porta-enxertos de marmeleiro, entre 6 a 8 anos após a enxertia, avaliadas durante os ciclos de cultivo 2011/12, 2012/13 e 2013/14.

\begin{tabular}{lcc}
\hline Combinações & Incompatibilidade 'translocada' & Incompatibilidade 'localizada' \\
\hline 'Abbè Fetel'/‘Adams' & $45,4 \mathrm{a}$ & $\mathrm{B}$ \\
'Clapp's Favourite'/'EMA' & $34,0 \mathrm{c}$ & $\mathrm{D}$ \\
'Decana du Comice'/‘Adams' & $41,2 \mathrm{~b}$ & $\mathrm{~B}$ \\
'Packham's Triumph'/‘EMA' & $46,0 \mathrm{a}$ & $\mathrm{B}$ \\
"Rocha'/‘Adams" & $44,8 \mathrm{ab}$ & $\mathrm{A}$ \\
$\mathrm{CV}(\%)$ & 4,4 & \\
\hline
\end{tabular}

*Médias seguidas pela mesma letra na coluna não diferem estatisticamente entre si pelo teste de Duncan a 5\% de probabilidade de erro. A: união perfeita; B: união boa; C: união com descontinuidade na casca; D: união com descontinuidade na casca e lenho; E: quebra da união.

Triumph'/‘EMA' apresentaram uma boa união na zona de enxertia (Figura 1), pois a linha de união na casca e no lenho são contínuas, embora no lenho seja, muitas vezes, visível, devido à excessiva formação de raias. Os vasos do xilema não formaram um conduto totalmente contínuo dos porta-enxertos com as cultivares pelo acúmulo do corante na região do enxerto,

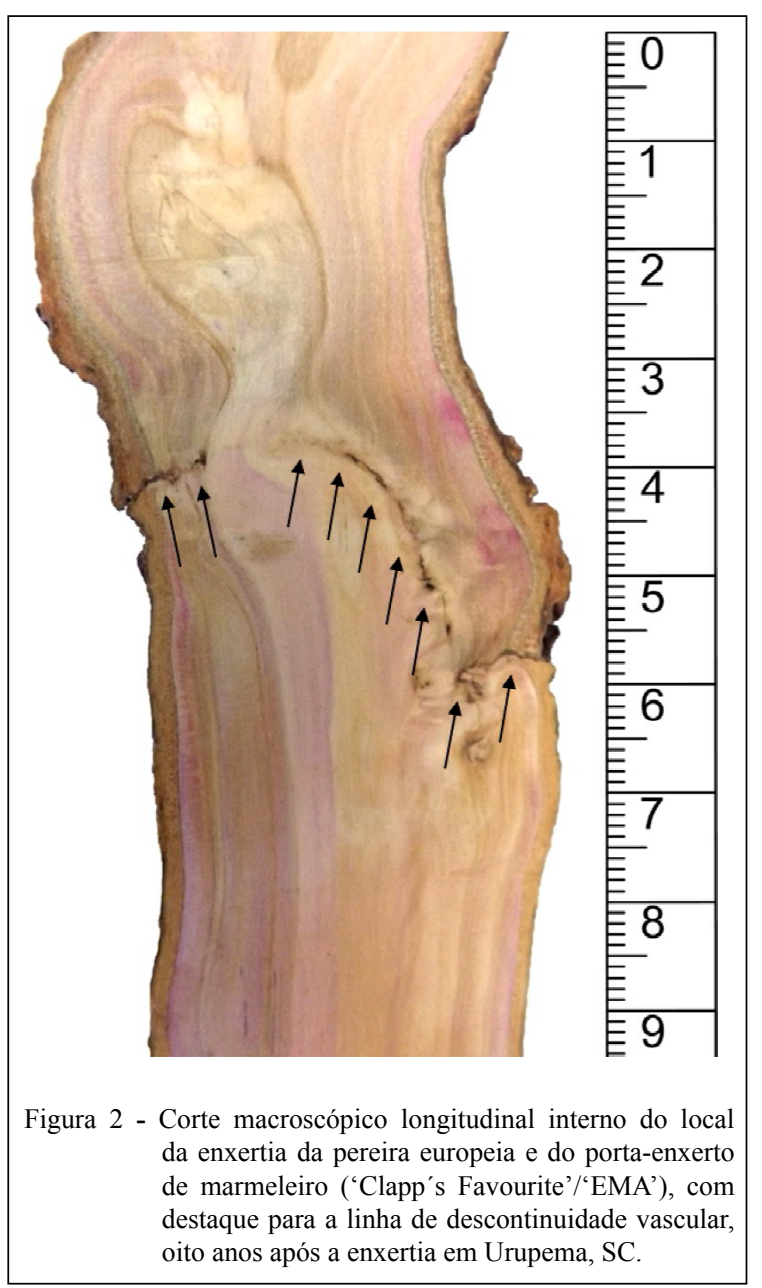

sendo as três combinações pertencentes à classe $\mathrm{B}$, a qual apresenta uma boa união e união com descontinuidade (Figura 1), porém sem prejuízos a resistência mecânica.

\section{CONCLUSÃO}

A combinação 'Clapp's Favourite'/'EMA' apresentou incompatibilidade do tipo 'localizada' pela descontinuidade vascular na região de união do enxerto. As combinações 'Santa Maria'/'Adams', 'Decana du Comice'/AAdams' e 'Packham's Triumph'/'EMA' apresentaram boa união na zona de enxertia pela continuidade da linha de união na casca e no lenho. A combinação 'Clapp's Favourite'/‘EMA', devido às regiões de incompatibilidade encontradas, não é uma combinação recomendada nas condições experimentais avaliadas. As combinações 'Abbè Fetel'/'Adams' e 'Rocha'/'Adams' são as mais indicadas para plantio, devido à total compatibilidade encontrada entre a cultivar e o porta-enxerto. As combinações 'Santa Maria'/'Adams', 'Decana du Comice'/'Adams' e 'Packham's Triumph'/'EMA' podem ser recomendadas devido à compatibilidade parcial de enxertia, sendo indicadas para alta e super densidades de plantio.

\section{AGRADECIMENTOS}

Os autores agradecem à Coordenação de Aperfeiçoamento de Pessoal de Nível Superior (CAPES), pelo apoio financeiro a este projeto.

\section{REFERÊNCIAS}

EPAGRI (EMPRESA DE PESQUISA AGROPECUÁRIA E EXTENSÃO RURAL DE SANTA CATARINA). Dados meteorológicos de São Joaquim e Videira, SC. Florianópolis: EPAGRI/CIRAM/INMET, 2013. Disponível em: <http://ciram. epagri.sc.gov.br/portal/website/index.jsp?url=jsp/monitoramento/ monitoramento.jsp\&tipo=rios $>$. Acesso em: 08 jun. 2012. 
FACHINELLO, J.C et al. Situação e perspectivas da fruticultura de clima temperado no Brasil. Revista Brasileira de Fruticultura, v.33, n.1, p.109-120, 2011. Disponível em: <http://www.scielo.br/ scielo.php?pid=S0100-29452011000500014\&script=sci_arttext $>$. Acesso em: 08 jun. 2014. doi: 10.1590/S0100-29452011000500014.

FAO. Faostat Database Prodstat. Disponível em: $<$ http://faostat fao.org/faostat/servlet/>. Acesso em: 25 mar. 2013.

GIACOBBO, C.L. et al. Compatibilidade entre o marmeleiro portaenxerto cv. 'EMC' e cultivares de pereira. Scientia Agraria, v.8, n.1, p.33-37, 2007. Disponível em: <http://ojs.c3sl.ufpr.br/ojs2/index. php/agraria/article/view/8339>. Acesso em: 08 jun. 2014.

MILOSEVIC, T.; MILOSEVIC, N. Influence of cultivar and Rootstock on early growth and syllepsis in nursery tress of pear (Pyruscommunis L., Rosaceae). Brazilian Archives Biology and Technology, v.54, n.3, p.451-456, 2011. Disponível em: <http:// www.scielo.br/scielo.php?pid=S151689132011000300004\&scr $\mathrm{ipt}=$ sci arttext $>$. Acesso em: 08 jun. 2014. doi: 10.1590/S151689132011000300004 .

MOSSE, B.; HERRERO, J. Studies on incompatibility between some pear and quince grafts. Journal Horticultural Science, v.26, p.238-245, 1951. Disponível em: <http://www.jhortscib.org/ members/showdocument?lidvan $=$ jhsb \&series $=$ jhsb\&vid $=26 \&$ iid $=3 \&$ article $=4 \&$ action $=$ showdocument $>$. Acesso em: 08 jun. 2014 .

MOSSE, B. Graft incompatibility in fruit trees. Horticultural Plant Crops, v.28, p.36-41, 1962. Disponível em: <http://www.fruticultura.org/teses/58/ TESE Ivan dos Santos Pereira.pdf?.1366073601>. Acesso em: 25 jul. 2014.

PINA, A.; ERREA, P. Morphological and histochemical features of compatible and incompatible stem unions. Acta
Horticulturae, v.814, p.453-456, 2009. Disponível em: <http:// www.actahort.org/members/showpdf?booknrarnr=814_77>. Acesso em: 08 jun. 2014

PEEL, M.C. et al. Updated world map of the Koppen-Geiger climate classification. Hydrology Earth System Science, v.11, p.1633-1644, 2007. Disponível em: <http://www.hydrol-earthsyst-sci.net/11/1633/2007/hess-11-1633-2007.pdf $>$. Acesso em: 24 out. 2014.

PIO, R. et al. Grafting of quince 'Portugal' on Cydonia and Chaenomeles rootstocks. Revista Brasileira de Fruticultura, v.30, n.3, p.850-852, 2008. Disponível em: $<$ http://www.scielo. br/scielo.php?pid $=\mathrm{S} 0100-29452008000300051 \&$ script $=$ sci arttext $>$. Acesso em: 08 jun. 2014. doi: 10.1590/S010029452008000300051

RODRIGUES, A.C. et al. Compatibilidade entre diferentes combinações de cvs. Copas e porta-enxertos de Prunus sp. Revista Brasileira Agrociência, v.10, p.185-189, 2004. Disponível em: $<$ http://periodicos.ufpel.edu.br/ojs2/index.php/CAST/article/ viewArticle/916>. Acesso em: 08 jun. 2014.

TECCHIO, M.A. et al. Evolution and perspective of the temperate fruit crops in São Paulo state, Brazil. Revista Brasileira de Fruticultura, v.33, p.150-157, 2011. Edição especial. Disponível em: $\quad<$ http://www.scielo.br/scielo.php?script=sci_arttext\&p $\mathrm{id}=$ S0100-29452011000500018>. Acesso em: 08 jun. 2014. doi: $10.1590 / \mathrm{S} 0100-29452011000500018$.

ZARROUK, O. et al. Graft compatibility for new peach rootstocks in nursery. Acta Horticulturae, v.713, p.327-330, 2006. Disponível em: <http://www.actahort.org/books/713/713 47. htm>. Acesso em: 08 jun. 2014. 